Cahiers de recherches médiévales

\title{
Bons et loyaux services
}

Les contrats d'apprentissage en Orléanais (1380-1480)

Françoise Michaud-Fréjaville

\section{(2) OpenEdition \\ Journals}

Édition électronique

URL : https://journals.openedition.org/crm/716

DOI : $10.4000 / \mathrm{crm} .716$

ISSN : 1955-2424

Éditeur

Honoré Champion

Édition imprimée

Date de publication : 1 juin 2005

Pagination : 11-30

ISSN : 1272-9752

Référence électronique

Françoise Michaud-Fréjaville, «Bons et loyaux services », Cahiers de recherches médiévales [En ligne],

12 spécial | 2005, mis en ligne le 28 juin 2008, consulté le 15 décembre 2022. URL : http://

journals.openedition.org/crm/716; DOI : https://doi.org/10.4000/crm.716 


\title{
rig
}

\author{
$-2-$ \\ Bons et loyaux services : \\ Les contrats d'apprentissage en Orléanais (1380-1480)*
}

«Plus seure richece est enrichir son filz par aucun mestier que se l'en li lessoit deniers ou possessions; que toute autre richece lesse l'ome fors que mestier. Pource fiuz, je te conseil que tu apreigne aucun mestier duquel tu peusses vivre se mestier estoit». Tel est le conseil que donne Raymond Lulle dans la Doctrine d'enfant ${ }^{1}$. Cet éloge du travail s'adresse aux riches comme aux pauvres puisque «maint homme lesse son fiuz riche, qui vient a povrete et a mort porce que il gaste et despent sa richece et ne set point de mestier dont il vive». Aussi l'enfant devrait-il apprendre un des «arts mechaniques [...] c'est assavoir labereors de terres, fevres, charpentiers, cordouaniers, marcheanz $»^{2}$. Mais l'apprendre où et comment? Par la cohabitation permanente avec ceux qui vivent d'un métier, par l'exemple et par la pratique, l'idéal étant que le père initie son fils à son art comme Joseph le fit pour Jésus à Nazareth. Mais cet exemple ne pouvait raisonnablement être toujours réalisé : il y avait souvent trop d'enfants pour qu'ils puissent ensemble relever le métier du père, ou bien, un des parents disparu, la famille était dispersée. Il arrivait que l'enfant eût le goût d'une autre activité, ou le désir de ne rien faire; des parents manifestaient parfois de l'ambition; le hasard menait un protecteur à envisager un autre avenir pour un enfant. Dans ces cas-là, l'apprentissage se faisait hors de la maison paternelle, et assez souvent fort loin. Il n'était d'ailleurs pas mauvais d'aller apprendre des tours de mains différents, des techniques locales ; il pouvait être salubre de se frotter à d'autres caractères. Nous ne saurons jamais la proportion de ceux qui apprirent au sein de leur maison ou dans les champs paternels les gestes qui leur permettraient d'exercer l'activité qui les nourrirait à l'âge adulte; en revanche, les contrats d'apprentissage permettent une certaine approche de ceux qui purent ou durent trouver ailleurs le cadre de leur éducation professionnelle.

La plupart des études sur le sujet se fondent sur l'analyse des statuts des différents métiers «réglés $»^{3}$. Elles confondent ainsi, en grande partie à cause d'une documentation encore inexploitée, un idéal - celui des promoteurs de ces règlements - avec la réalité quotidienne de la pratique. Ferait-on une Histoire de l'éducation au $\mathrm{XX}^{\mathrm{e}}$ siècle avec le seul Bulletin Officiel de l'Éducation Nationale?

*Les références sont celles des Archives départementales du Loiret.

${ }^{1}$ R. Lulle, Doctrine d'enfant, éd. A. Llinarès. Paris, 1969, p. 170.

${ }^{2}$ Ibid., p. 169.

${ }^{3}$ E. Dolléans, Histoire des métiers et formations professionnelles dans la société française depuis le XIII siècle, Paris, 1943 ; P. Quef, Histoire de l'apprentissage, aspect de la formation technique et commerciale, Paris, 1964. G. Fagniez, Etudes sur l'industrie et la classe industrielle à Paris aux XIII et XIV siècles, Paris, 1877, et A. Gouron, La réglementation des métiers en Languedoc, Paris, 1958, ont, pour leur part, très bien senti qu'il fallait chercher audelà des statuts de métiers.

Cahiers de Recherches Médiévales (XII - XV $V^{e}$ s.), 12spé, 2005 
Certains aspects de la pratique se trouvent, au prix d'une pêche qui n'est pas toujours miraculeuse, dans la masse des archives judiciaires ${ }^{4}$ et dans les dépôts des notaires. L'Orléanais ayant la chance de disposer de très belles séries notariales remontant au XIV ${ }^{\mathrm{e}}$ siècle, il était tentant d'essayer de mener une recherche quantitative sur les contrats d'apprentissage.

Cette enquête en est à sa seconde étape. Un premier dépouillement a été effectué en 1978-1979 pour la période 1380-1440. Il est pratiquement exhaustif quant au matériau utilisable, 376 contrats pour les villes d'Orléans, Beaugency, Jargeau et Meung-sur-Loire ${ }^{5}$; des résultats partiels en ont été publiés ${ }^{6}$. La période suivante est beaucoup plus riche ${ }^{7}$, malgré la destruction des dépôts d'avant 1940 ; il existe cependant un trou relatif pour la période 1430-1445 dont ne subsistent que des épaves. Un second dépouillement a été effectué, grandement facilité par les répertoires conservés, pour quelques années seulement, de trois notaires orléanais (Garsonnet, Prevost, Noblet), ce qui a permis d'étudier 439 contrats répartis dans 26 registres échelonnés entre 1435 et $1489^{8}$. À cela se sont ajoutés deux registres balgenciens $(1431,1449-1450)^{9}$.

Une troisième étape consistera à relever systématiquement les contrats des notaires orléanais jusqu'en 1500, à effectuer des sondages pour les autres villes et mettre au point un programme informatique afin de traiter vraisemblablement plus de 2.000 contrats. Cependant les inégalités d'étude à étude et l'état de conservation des documents ne permettent pas de faire des estimations sérieuses sur ce matériau à venir: tel notaire ne donne pas un contrat entre 1433 et $1438^{10}$, quand un autre en fournit 42 pour la seule période 1434-1437'11.

Les sources dont nous disposons à l'heure actuelle permettent de couvrir une centaine d'années, depuis la fin du règne de Charles $\mathrm{V}$ jusqu'aux dernières années de Charles VIII. Le duché a vécu au cours de ce siècle une crise extrêmement grave entre 1407 et 1430, étant le cœur du domaine «armagnac»; puis, comme le reste du royaume, il a connu une période de reconstruction et de relative prospérité. Dans le cadre rigide d'un acte notarié, peut-on percevoir une évolution séculaire de la relation entre le maître et l'apprenti? Y a-t-il eu modification des caractéristiques de l'une ou l'autre des parties? Aux injonctions apparemment impératives des statuts, la réponse de la pratique fut-elle ferme ou souple? Le contrat d'apprentissage est-il

${ }^{4} \mathrm{~B}$. Geremek, Le salariat dans l'artisanat parisien aux XIII $-X V^{e}$ siècles, Paris, 1968, p. 11 : «Derrière la sévère ordonnance de l'édifice représenté par les statuts des corporations, les actes judiciaires nous montrent l'aspect humain, la forme quotidienne des rapports sociaux ». ${ }^{5}$ Beaugency : 3 E 1163 à 1180 (1397-1451); Meung-sur-Loire : 3 E 8433 (1418-1432); Orléans : 3 E 10124 à 10144 (1385-1437); Jargeau : 3 E 14321 à 14330 (1381-1423).

${ }^{6} \mathrm{~F}$. Michaud-Fréjaville, «Les enfants au travail, contrats d'apprentissage en Orléanais (13801450) », dans L'enfant au Moyen Âge, Senéfiance, n ${ }^{\circ}$, Aix-en-Provence, 1980, p. 63-71.

'Jusqu'en 1501, on dispose de 27 registres pour Jargeau, 47 pour Beaugency, 21 pour Meung, 98 pour Orléans.

${ }^{8}$ Dépôt Fauchon, 3 E 10159 à 3 E 10165 (inventaire 3 E 10291), 3 E 10210 à 3 E 10212 (inventaire 3 E 10292), 3 E 10224 à 3 E 10338 (inventaire 3 E 10293).

${ }^{9} 3$ E $627-628$.

${ }^{10} 3$ E 10150, Orléans, Jean de Recoin.

${ }^{11} 3$ E 10137-10138, Orléans, Christophe. 
une source «fiable» de la connaissance des travailleurs médiévaux ? Les réflexions que je propose, fondées sur le quantitatif, ne sont qu'une amorce de réponse.

\section{Les contrats}

«Perrin Thierri, marchant, demorant a Blois, baille Perrin Thierri son fils, de l'aage de quinze ans ou environ, lequel en la presence et du consentement et auctorité dudit Perrin son pere [...] s'est semblablement baillé, alloé et accueilli a Jehan de Mondidier dit Pavé, marchand changeur et bourgois d'Orlians [...] pour estre et demourer avec lui du jour de la Toussains [...] jusques a trois ans [...] et le servir ou fait de son change [...] et en toutes choses licites et honnestes et leaument ainsi que serviteur apprenti doit servir son maitre ; et avec ce ledit $[. .$.$] pere [. .$.$] paiera [. .$.$] la$ somme de vint reaux d'or [...] et se le dit Pavé trouve faulte en son change [...] le dit Perrin sera tenu le lui rendre et restituer; moiennant [...] que ledit Pavé lui sera tenu querir et admenistrer boire, manger, feu, lit et hostel et sa chaussement de solier [...] et lui apprendre a son povoir la science et marchandise dont il s'entremet» $(8 . I X .1437)^{12}$. Ces quelques lignes d'un contrat tout à fait banal permettent l'établissement d'une liste-type des renseignements exploitables : nom, âge, origine (lieu de naissance ou de résidence), existence ou décès des parents, profession du père, qualités de ceux qui présentent l'apprenti, tous éléments qui permettent de trouver les caractéristiques du groupe des impétrants. Viennent ensuite les composantes de l'apprentissage: nom, métier, localisation du maître, durée prévue du contrat, clauses financières et morales. Enfin, d'éventuelles mentions marginales donnent la longueur effective du séjour et les modifications intervenues après la conclusion de l'accord. En tout, une quinzaine de données sont ainsi théoriquement utilisables. Il est bien évident que tous les actes dont nous disposons n'offrent pas l'ensemble de ces renseignements; il manque assez souvent le lieu d'origine et le métier du père parfois la mention même des parents ; plus rarement ne figure pas l'âge de l'apprenti; enfin les mentions marginales sont hélas très rares (elles ne se rencontrent que dans $5 \%$ environ des contrats).

Notre corpus se répartit ainsi :

TABLEAU I

Répartition des contrats

\begin{tabular}{|l|l|l|l|}
\hline & Garçons & Filles & Total \\
\hline $1380-1440$ & 337 & 40 & 377 \\
& $89,3 \%$ & $10,7 \%$ & $100 \%$ \\
\hline $1440-1490$ & 428 & 16 & 444 \\
& $96,4 \%$ & $3,6 \%$ & $100 \%$ \\
\hline Total & 765 & 56 & 821 \\
& $93,2 \%$ & $6,8 \%$ & $100 \%$ \\
\hline
\end{tabular}

${ }^{12} 3$ E 10151. 
Une écrasante priorité apparaît ainsi accordée au travail des garçons; l'apprentissage par contrat est un mode d'entrée dans le monde des gens de métier fondamentalement masculin. Cependant d'assez nombreuses femmes, si l'on en croit les listes d'imposition, exerçaient semble-t-il un métier ${ }^{13}$, Bien évidemment, nombre d'entre elles l'avaient appris en côtoyant quotidiennement l'activité de l'atelier de leur père ou de leur époux, et de fait on s'aperçoit que les femmes citées dans les listes des gens de métier sont la plupart du temps des veuves ${ }^{14}$, Cela ne pouvait concerner toutes les fillettes d'âge actif; nous devons donc supposer que l'on ne ressentait pas la nécessité de dépenser, afin de protéger l'apprentissage féminin, la somme nécessaire à l'établissement d'un acte notarié. Si faible soit-il au début du XV siècle, le nombre de ces contrats diminue encore au cours de la seconde moitié du siècle ${ }^{15}$; l'échantillonnage n'est pas suffisant pour pouvoir pousser très loin les explications. Tout au plus peut-on supposer que lors de périodes démographiquement difficiles, telles les premières décennies du $\mathrm{XV}^{\mathrm{e}}$ siècle, les jeunes filles ou leurs ayants droit pouvaient parfois exiger de celui qui accueillait une future lingère ou une chambrière, les garanties offertes par un contrat.

\section{Des jeunes sans foyer?}

En feuilletant rapidement les actes, un élément frappe d'emblée: la grande fréquence de la mention du décès d'un ou des deux parents. Ce phénomène répétitif paraît amplifié encore par la présence dans certains registres des actes de mise en tutelle, actes dont la formulation est très voisine de celles de nos contrats. Les tuteurs placent auprès de $\mathrm{X}$... un enfant pour $\mathrm{X}$ années ; le «preneur» lui fournira toutes ses nécessités «comme boire, manger, vestir, chausser, giste, feu et heberger». L'enfant peut devoir servir celui qui le prend «en toutes ses besognes licites et raisonnables $»^{16}$. Tutelles et apprentissages sont chez certains notaires signalés en marge par la même mention «loage d'enfant». apprentis :

Les chiffres corroborent en fait la fréquence des orphelins engagés comme

${ }^{13}$ Une courte liste de métiers féminins est donnée par K. Michaë1sson, La taille de Paris en 1313, Göteborg, 1951, p. XVIII-XIX. Il relève, outre les chambrières : huilière, chandelière, regratière, hôtelière, chasublière.

${ }^{14} \mathrm{Il}$ subsiste pour Orléans quelques listes de gens de métier: en 1385, sur 25 tisserands en linge, on trouve une femme, Jehanne la Tillette, veuve de Perot Moynart (A 2196 fol. 2v) ; les listes postérieures ne comportent que des hommes. Il faut cependant noter que n'y figure souvent que la «melior et saine» partie des maîtres; on peut penser que les femmes, qui ne faisaient que l'intérim entre leur mari et leur fils, n'étaient pas consultées lors des assemblées. ${ }^{15} \mathrm{R}$. Mousnier, La famille, l'enfant et l'éducation en France et en Grande-Bretagne, Paris, 1975, p. 152, dit qu'à cette époque, en Angleterre, il y avait beaucoup de filles en apprentissage. Je demeure sceptique.

${ }^{16} 3$ E 1174, 13-II-1452, tutelle d'Etienne Dolpest, placé pour 6 ans près de Laurent Biergaut. 


\section{TABLEAU II}

Répartition des orphelins par sexe

\begin{tabular}{|l|l|l|l|}
\hline & Garçons & Filles & Total \\
\hline $1380-1440$ & 213 & 25 & 238 \\
\hline $1440-1490$ & 245 & 11 & 256 \\
\hline Total & 458 & 36 & 494 \\
\hline
\end{tabular}

Si l'on rapporte ces chiffres au total des apprentis, on constate que $60,2 \%$ de ceux qui se présentent pour vivre chez un maître ont quitté un foyer familial qui n'était plus conjugal. Il faut donc se garder de l'image du fils «abandonné » par une famille quasiment indifférente : dans de nombreux cas, on ne pouvait tout simplement pas le garder. Le tableau II montre que le poids des orphelins doit être modulé dans le temps. Il est plus important avant 1440 (63\%), qu'au cours de la période suivante $(57 \%)$. Une explication paraît de prime abord évidente : répercussion des troubles du début du $\mathrm{XV}^{\mathrm{e}}$ siècle, une mortalité accrue a laissé à eux-mêmes, ou à des tuteurs, des jeunes déjà déracinés.

Il me semble nécessaire d'apporter des nuances à cette explication : le contrat est un type particulier d'accord entre individus. Il a pour lui d'être une preuve opposable judiciairement, il a contre lui de n'être rédigé qu'à titre onéreux. Dans la mesure où les orphelins sont placés sous l'autorité d'une tutelle, les curateurs doivent se prémunir contre d'éventuelles demandes de compte en allant chez le notaire. D'autre part, un jeune venant se présenter seul à un maître qui va exercer sur lui une autorité de type paternel, a tout intérêt à préciser si la mort de ses parents lui permet de disposer de lui-même, et, éventuellement, d'un héritage. Ainsi nous n'aurions qu'une source faussée dès l'origine et qui ne reflèterait que le caractère protégé de l'orphelin, au lieu de nous livrer un échantillonnage représentatif de l'ensemble des apprentis. La coutume protégeait les enfants sans parents en leur donnant des tuteurs et en tentant de les garantir des mauvais avoués : «l'en octroie tant seulement à ces personnes à avoer orfelins à cels qui par nature ou par debonereté avoent et de toz autres l'en doit l'en deffendre ${ }^{11}$. La royauté puis les ducs assuraient d'ailleurs la protection des biens et de la personne : « Li baillif s'en orra [...] de cels qui ont par loier les baus des orfelins, et de cels qui porchacent que li orfelins aient mauvais tutor, et de cels qui par lor conseils font que l'orfelin maumet ses biens ${ }^{18}$.

Au cours de la seconde moitié du siècle, l'embauche se faisant plus large, les maîtres ne pouvaient pas demander autant de garanties. Enfin, plus l'apprenti était âgé, plus il risquait évidemment d'avoir perdu l'un ou l'autre de ses parents, mais alors cela ne peut plus sous-entendre une mise en apprentissage autoritaire afin de se «débarrasser» d'un enfant d'un premier lit, ou d'une bouche inactive à nourrir. On avait affaire à de jeunes adultes, capables d'initiatives, susceptibles de discuter des clauses de leur contrat. Leur qualité d'orphelin renforçait leur indépendance, justifiait leur demande de faire procéder à un contrat leur garantissant les prestations du

${ }^{17}$ Li Livres de Jostice et de Plet, éd. Rapetti, Paris, 1850, p. 61.

${ }^{18}$ Ibid., p. 69. 
maître, expliquait, et moralisait dans une certaine mesure, le voyage qui les avait menés de tous les horizons du royaume jusqu'aux échoppes des bords de Loire.

Tel qu'il nous est donné, le groupe des orphelins se présente avec une étonnante caractéristique : l'écrasante prédominance du décès paternel.

TABLEAU III

Ventilation des orphelins en fonction du parent décédé

\begin{tabular}{|l|l|l|l|l|l|}
\hline & & $\begin{array}{l}\text { Orphelins } \\
\text { de père }\end{array}$ & $\begin{array}{l}\text { Orphelins } \\
\text { de mère }\end{array}$ & $\begin{array}{l}\text { Orphelins de père } \\
\text { et mère }\end{array}$ & Total \\
\hline $\begin{array}{l}1380- \\
1440\end{array}$ & Filles & 13 & 8 & 4 & \\
\hline & Garçons & 154 & 30 & 29 & 238 \\
\hline & Total A & 167 & 38 & 33 & \\
\hline $\begin{array}{l}1440- \\
1480\end{array}$ & Filles & 5 & 4 & 2 & 256 \\
\hline & Garçons & 152 & 45 & 48 & 494 \\
\hline & Total B & 157 & 49 & 50 & 83 \\
\hline & $\begin{array}{l}\text { Total A } \\
+ \text { B }\end{array}$ & 324 & 87 & 83 & \\
\hline
\end{tabular}

Ainsi $83 \%$ des orphelins se trouveraient sans père, et ce taux rapporté à l'ensemble des garçons ferait que pour près de la moitié des contrats $(50,06 \%)$, le maître remplacerait véritablement le père. Cette proportion est ici très importante, deux fois ce qui a été constaté pour Tours en 1473-1477'. L'explication est-elle démographique? Peut-on y voir par exemple les conséquences du mariage plus tardif des hommes ? Pour ma part, je pense que la raison en est davantage sociologique : la sur-mortalité masculine est une sous-déclaration du décès maternel. La mention du père, même défunt, sert à asseoir le jeune qui veut se placer au sein d'un espace familial et territorial. Aucun contrat ne donne comme référence la seule mention d'une mère disparue. Les remariages maternels sont souvent indiqués, le beaupère sert alors à fournir, au déclarant et à sa propre femme, l'assise sociale et spatiale qui permet d'insérer l'apprenti dans un cadre rassurant pour le maître. De façon générale, la mort de la mère n'est signalée que si elle a une incidence financière (héritage permettant un versement au patron), ou légale (minorité incapable). Nous n'avons malheureusement aucun moyen de pondérer la sur-représentation des orphelins de père. Notre source est ici tout à fait non-objective.

\section{Enfants au travail ?}

Cette même non-objectivité du corpus me semble se retrouver dans la présentation des âges des apprentis. La mention de l'âge est une des données les plus précieuses des contrats; hélas! sur ce point, la conscience professionnelle ou les

${ }^{19} \mathrm{P}$. Le Bourgeois, L'apprentissage à Tours de la seconde moitié du XVe siècle au début du XVI $I^{e}$ siècle (mém. maîtrise, dact.), Tours, 1969-1970, p. 19: $25 \%$ d'orphelins de père en $1473-1477,38 \%$ en $1518-1520$. 
habitudes des clercs de notaire ont joué. Dans un quart des cas, l'âge ne nous est pas donné ; cependant, Maître Noblet et Maître Prevost devaient avoir donné l'ordre de faire préciser cet élément puisque, de 1440 à 1489, près de $93 \%$ des contrats en font cas. A la fin du XIV et au début du XV $\mathrm{XV}^{\mathrm{e}}$ siècle néanmoins, des indications permettent une évaluation des âges si celui-ci est omis. Les apprentis sont «mineurs" quand est indiqué «se obligera quand il sera suffisamment agé»; inversement ils sont «majeurs » quand figurent des formules du type «suffisamment agé, si comme il disoit, et comme il apparoit par l'examen de sa personne $»^{20}$, ou encore quand on prévoit pour eux, en cas de rupture de contrat, de les mettre en prison «fermée». En fait, ce qui apparaît comme fondamental est la notion de majorité ou de minorité, d'où l'intérêt particulier présenté par le groupe des 13-15 ans.

Y a-t-il un âge de l'apprentissage ? Nos «alloués»s'échelonnent entre trois $a^{21} s^{21}$ et 24 ans $^{22}$, mais ces extrêmes sont aberrants. L'ensemble des contrats concerne des individus de 7 à 20 ans. J'ai distingué trois classes pour les garçons, la minorité certaine des moins de 13 ans, la majorité assurée des plus de 16, et entre les deux la période cruciale des 14-15 ans. La coutume de l'Orléanais n'est pas sur cette question d'une éblouissante clarté : elle protège «ceulx qui n'ont quinze ans [...] quar emprès seize ans, ils sont en aage de vigor $»^{23}$. Les contrats cependant précisent pour des mineurs qu'ils s'obligeront quand ils auront $14 \mathrm{ans}^{24}$. La répartition de ces groupes donne les chiffres suivants :

TABLEAU IV

Âge des garçons au contrat

\begin{tabular}{|l|l|l|l|l|}
\hline & -13 ans & $13-15$ ans & +16 ans & Total \\
\hline $1380-$ & 39 & 86 & 86 & 211 \\
1440 & $(18,4 \%)$ & $(40,8 \%)$ & $(40,8 \%)$ & $(100 \%)$ \\
\hline $1440-$ & 40 & 144 & 217 & 401 \\
1490 & $(10 \%)$ & $(35,9 \%)$ & $(54,1 \%)$ & $(100)$ \\
\hline Total & 79 & 230 & 303 & 612 \\
& $(12,9 \%)$ & $(37,6 \%)$ & $(49,5 \%)$ & $(100)$ \\
\hline
\end{tabular}

\footnotetext{
${ }^{20}$ Cette belle formule ne trouverait-elle pas sa raison d'être dans le désir des clercs de tirer à la ligne pour se faire payer plus cher?

${ }^{21} 3$ E 10237, 27-XII-1487 : Pierre Garraut, fils de feu Pierre Garraut, placé par ses parents et tuteurs chez un vigneron. Contrat de 15 ans (!), au cours duquel on lui enseignera le « fait de vignerie "; si l'enfant meurt, ses frères seront dispensés de payer en entier l'apprentissage.

${ }^{22} 3$ E 10236, 10-VII-1486 : Jehan Le Chandelier. Il ne s'agit pas d'une embauche, il se place chez un tondeur de drap pour deux ans et paie 2 écus d'or; un de ses oncles, orfèvre, se porte garant du versement.

${ }^{23}$ Li livres..., op. cit., p. 116.

${ }^{24} 3$ E 10138, 8-IV-1439 : Noël Cousin, 12 ans « sera tenu de se obliger quant il aura quatorze ans $»$.
} 
Quant aux filles, leur majorité est assurée à 12 ans $^{25}$, d'où un décalage nécessaire des classes d'âge.

\section{TABLEAU V}

Âge des filles au contrat

\begin{tabular}{|l|l|l|l|l|}
\hline & -12 ans & $12-13$ ans & +13 ans & Total \\
\hline $1380-1440$ & 15 & 2 & 4 & 21 \\
\hline $1440-1490$ & 9 & 2 & 1 & 12 \\
\hline Total & 24 & 4 & 5 & 33 \\
\hline
\end{tabular}

Les 612 mentions d'âge des garçons permettent d'établir l'histogramme des âges d'entrée en apprentissage :

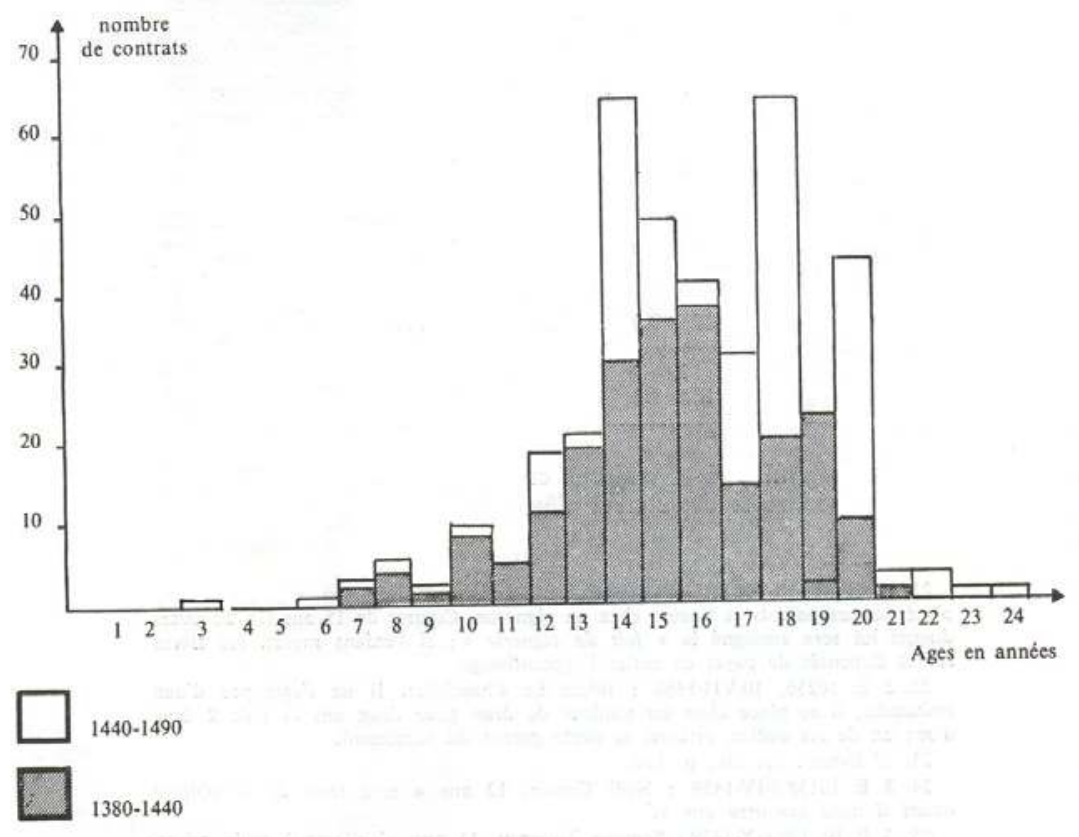

I : Age des garçons à l'embauche

Histogramme et tableaux ébranlent une des données traditionnelles de l'apprentissage : 14 ans n'est pas l'âge normal de l'entrée dans le monde du travail, il n'est même pas l'âge moyen (15 ans et demi).

$\mathrm{Au}$ cours de la seconde moitié du $\mathrm{XV}^{\mathrm{e}}$ siècle, les débuts dans la vie professionnelle se font, pour les garçons, bien après la majorité. La moyenne d'âge de la

${ }^{25} 3$ E 10 139, 6-X-1439 : Symone Tonneau, 11 ans, s'obligera à tenir prison fermée quand elle aura douze ans, si elle abandonne son apprentissage. 
troisième classe, celle des plus de 16 ans (en éliminant les 23-24 ans), est de 18 ans, l'histogramme reflétant la coïncidence du mode et de la moyenne, dans ces conditions, il ne s'agit plus du tout d'enfants ${ }^{26}$. Cela est d'autant plus net que nous disposons pour cette période de la quasi-totalité des âges. Le problème est légèrement différent pour le demi-siècle précédent au cours duquel les appréciations tiennent lieu de chiffres. Comme ces expressions «suffisamment aagé » ou «mineur d'aage» se rapportent aux groupes des 13-15 ans et des moins de 13 ans, il me semble que lorsque aucune indication ne figure, le contrat concerne un jeune adulte (plus de 16 ans) dont il était évident aux yeux de tous qu'il pouvait légalement s'engager. Ainsi s'expliquerait la différence de 14,6\% de la colonne des + 16 ans du tableau IV.

Les contrats d"apprentissage, utilisés comme source, et leurs données quantitatives, privilégient un groupe particulier, composé ici de ceux qui doivent faire la preuve qu'ils sont d'âge à assumer entièrement leur engagement d'apprentissage. Auraient-ils été aussi nombreux s'il avait été impossible de faire un apprentissage sans contrat? Je pense que l'on a trop tendance à confondre la majorité avec l'âge d'apprentissage ${ }^{27}$. L'entrée dans la vie active devait se faire plus tard qu'on ne le dit trop souvent en s'apitoyant sur la dureté des temps révolus. En fait, les plus jeunes de nos apprentis appartiennent à la catégorie privilégiée de ceux qui bénéficient d'un contrat.

Cela ne nous donne malheureusement pas d'aperçu sur ceux dont l'engagement était oral ; cela ne fait que raviver notre regret de ne pas savoir à quoi étaient occupés les enfants et les adolescents avant d'entrer dans la maison d'un maître ${ }^{28}$.

Le cas des filles est sans doute plus simple: pour elles, la douzième année marque tout bonnement leur effacement des contrats, pour autant que nous puissions juger sur un aussi faible effectif. Il paraît néanmoins évident que les documents dont nous disposons étaient destinés à protéger des mineures, orphelines de surcroît ${ }^{29}$. Lorsqu'elles étaient plus âgées, on ne jugeait plus nécessaire de faire la dépense du notaire...

\section{Isolement ou réseau de garanties?}

La plupart des contrats $(92,3 \%)$ contiennent une formule permettant de déterminer si l'impétrant s'est présenté seul devant le notaire ou s'il était accompagné d'un ayant droit ou d'un garant. Il ne s'agit pas ici de témoins, notre source

${ }^{26} \mathrm{C}$ 'est pourquoi j'émets des réserves sur des phrases comme : «dans l'artisanat parisien le plus souvent l'apprenti est encore un enfant : l'apprentissage commençait [...] en règle générale à 12 ans », (B. Geremek, Le salariat..., p. 54) dans la mesure où n'apparaît aucune donnée chiffrée. B. Chevalier, en s'appuyant sur 317 contrats a bien vu qu'à la fin du $X V^{\mathrm{e}}$ siècle, l'entrée en apprentissage se faisait à 16 ans (B. Chevalier, Tours, ville royale, Paris, 1975, p. 409).

${ }^{27}$ A. Gouron, op. cit., p. 268-269, remarque également le rôle de la minorité pubère (14 à 25 ans) dans le recrutement des apprentis, mais il n'a pas disposé de suffisamment d'indications d'âge pour aller plus loin. La même lacune dans la rédaction des contrats (et sur 1100 actes!) est déplorée par P. Wolff, Commerces et marchands de Toulouse, Paris, 1954, p. 434.

${ }^{28}$ Cette question est également soulevée par B. Chevalier, op. cit., p. 410.

${ }^{29} 75 \%$ des orphelines dont l'âge nous est donné ont moins de 13 ans. 
n'indique ces derniers que de façon rarissime ${ }^{30}$, mais de ceux qui ont la responsabilité ou l'initiative de l'engagement.

Le tableau ci-dessous résume cette donnée. J'ai fait figurer dans la colonne «mère " les contrats où la mère, veuve et non remariée, est seule à donner son consentement. Même dans ce cas nous ne savons que son prénom; la mention éventuelle de la paroisse d'origine est toujours rattachée au père décédé.

TABLEAU VI

Répartition des contrats en fonction de la personne qui présente l'apprenti

\begin{tabular}{|l|l|l|l|l|l|}
\hline & $\begin{array}{l}\text { Apprenti } \\
\text { présenté par }\end{array}$ & & & & Total \\
\hline & Le père & La mère & Un tiers & Lui-même & \\
\hline $1380-1440$ & 95 & 67 & 92 & 81 & 335 \\
\hline $1440-1490$ & 105 & 47 & 118 & 152 & 422 \\
\hline Total & 200 & 114 & 210 & 233 & 757 \\
& $(26,4 \%)$ & $(15,1 \%)$ & $(27,7 \%)$ & $(30,8 \%)$ & $(100 \%)$ \\
\hline
\end{tabular}

Dans $70 \%$ des cas, le maître était donc rassuré par la présence d'un adulte sur le sérieux de l'engagement, et inversement, l'apprenti recevait l'assurance, devant ses proches, d'un comportement honnête du maître. Il est vrai que, dans certains cas, la pression des adultes pouvait expliquer que l'adolescent se soit senti contraint et ait eu des velléités de fuite. Près de cinquante contrats prévoient cette éventualité (histogramme II). On constate alors que les «preneurs » ont exigé cette clause justement pour la classe d'âge douteuse des 13-16 ans, ne voulant point se voir opposer l'argument de la majorité rendant libre les jeunes de la disposition de leur corps.

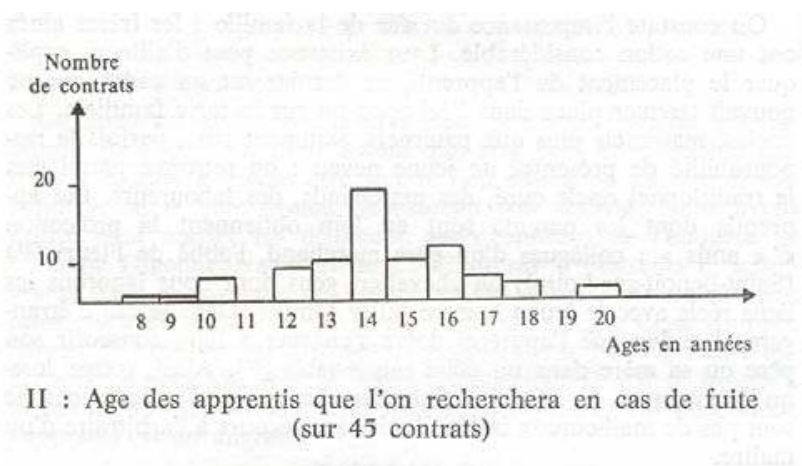

Le tableau VI montre que moins de la moitié des jeunes sont présentés par leur père ou leur mère; deux parents qui présentent ensemble leur fils est un cas de

${ }^{30} 3$ E 10162, 24-VIII-1464 : deux maitres du métier témoignent que le contrat sera profitable à Etienne Gorin, 14 ans, qualifié de mineur. 
figure qui ne se trouve que sept fois (il est comptabilisé dans la colonne «père »). La mère s'efface souvent devant son second mari, le «sire» de l'alloué, qui figure alors dans la colonne «tiers ». Le rôle des orphelins est souligné par la quasi-équivalence numérique des pères et des tiers : les tuteurs interviennent souvent en lieu et place du parent survivant.

Que pouvons-nous savoir des tiers qui sont à l'origine d'une mise en apprentissage ? Ils sont souvent en nombre : curateurs, amis, famille, surtout dans le cas des jeunes orphelins. Nous les avons regroupés par catégorie et non par individus, d'où une légère différence dans les totaux des tableaux VI et VII

TABLEAU VII

Répartition des tiers présentant un apprenti

\begin{tabular}{|l|l|l|l|l|}
\hline & Tuteurs & Famille & «Amis » & Total \\
\hline $1380-1490$ & 69 & 127 & 32 & 228 \\
\hline$\%$ & $30,3 \%$ & $55,7 \%$ & $14 \%$ & $100 \%$ \\
\hline
\end{tabular}

On constate l'importance du rôle de la famille : les frères aînés ont une action considérable. Leur présence peut d'ailleurs expliquer le placement de l'apprenti, ce dernier est un cadet qui ne pouvait trouver place dans l'échoppe ou sur la terre familiale. Les oncles, maternels plus que paternels, assument aussi parfois la responsabilité de présenter un jeune neveu: on retrouve parmi eux le traditionnel oncle curé, des marchands, des laboureurs. Les apprentis dont les parents sont au loin obtiennent la protection d' «amis»: collègues d'un père marchand, l'abbé de Fleury ${ }^{31}$ (SaintBenoît-sur-Loire), un chevalier, gens dont nous ignorons les liens réels avec le jeune homme ou sa famille. Dans le cas d'étrangers présentés par ces tiers, il arrive que l'apprenti doive s'engager à faire consentir son père ou sa mère dans un délai raisonnable $^{32}$. Ainsi, même lorsqu'ils viennent de contrées éloignées, beaucoup de ces jeunes ne sont pas de malheureux isolés, livrés sans recours à l'arbitraire d'un mâ̂tre.

Il y a pourtant des garçons qui se présentent seuls. C'est enfoncer une porte ouverte que de constater que ce sont les plus âgés ${ }^{33}$. Certes ils sont capables de se défendre ou de s'enfuir sans qu'on puisse réellement les pourchasser, mais le maître en les prenant ne risque pas grand-chose. Le plus souvent ces candidats viennent de loin, et il est prévu de leur verser un salaire en fin de service; si on leur rend la vie impossible, personne ne les défendra ; s'ils se sauvent, le salaire reste au patron.

${ }^{31} 3$ E 10338, 22-I-1489 (n. st.) : l'abbé de Fleury fait montrer le métier de barbier-chirurgien à un de ses serviteurs âgé de 18 ans.

${ }^{32} 3$ E 10216, 9-III-1465 (n. st.) : Jehan de Bonnes «a promis faire avoir agreable le présent contrat aux tuteurs dudit apprantiz ».

${ }^{33} 54 \%$ des apprentis de plus de 16 ans se présentent seuls pour signer le contrat. Bien enten$\mathrm{du}$, je refuse de considérer les $46 \%$ qui restent comme des «simples d'esprit ou des interdits ", ainsi que le suggère P. Didier pour les apprentis âgés présentés par un père ou un parent (P. Didier, «Le contrat d'apprentissage en Bourgogne aux XIV et $\mathrm{XV}^{\mathrm{e}}$ siècles », Revue Historique du droit français et étrangers, 1976, n 1, p. 38). 


\section{TABLEAU VIII}

Âge des apprentis se présentant seuls

\begin{tabular}{|l|l|l|l|l|}
\hline & -13 ans & $13-15$ ans & +16 ans & Total \\
\hline $1380-1440$ & 1 & 13 & 39 & 53 \\
& $(1,9 \%)$ & $(24,5 \%)$ & $(73,6 \%)$ & $(100 \%)$ \\
\hline $1440-1490$ & 2 & 15 & 125 & 14 \\
& $(1,4 \%)$ & $(10,5 \%)$ & $(88,1 \%)$ & $(100 \%)$ \\
\hline Total & 3 & 28 & 164 & 195 \\
& $(1,5 \%)$ & $(14,3 \%)$ & $(84,2 \%)$ & $(100 \%)$ \\
\hline
\end{tabular}

\section{Jeunesse déracinée?}

Un assez grand nombre d'actes précisent l'origine géographique de l'apprenti, pour autant que l'on pose en préalable que cette « origine » puisse être soit le lieu avoué de naissance, soit l'endroit où le père exerce ou exerçait son activité. Nous avons utilisé pour classer les distances les définitions de l'enquête d'A. Higounet-Nadal et de $\mathrm{Cl}$. Billot sur Migrants et immigrants dans le royaume de France, 1300-1550: est un migrant celui qui a dû se déplacer de plus de $30 \mathrm{~km}$. Cette distinction permet de répartir nos origines en trois groupes: I: le lieu d'apprentissage coïncide avec le lieu d'origine ${ }^{34}$; II : il se trouve à moins de $30 \mathrm{~km}$; III : il se situe à plus de $30 \mathrm{~km}$, l'apprenti est un alors un migrant.

TABLEAU IX

Origine géographique des apprentis

\begin{tabular}{|l|l|l|l|l|}
\hline & $\begin{array}{l}\text { I : Orig. de la ville } \\
\text { d'apprentissage }\end{array}$ & $\begin{array}{l}\text { II : Vient de } \\
\text { moins de 30 km }\end{array}$ & $\begin{array}{l}\text { III : Vient de } \\
\text { plus de 30 km }\end{array}$ & Total \\
\hline $1380-$ & 66 & 88 & 75 & 229 \\
1440 & $(28,8 \%)$ & $(38,5 \%)$ & $(32,7 \%)$ & $(100 \%)$ \\
\hline $1440-$ & 93 & 43 & 217 & 353 \\
1490 & $(26,3 \%)$ & $(12,2 \%)$ & $(61,5 \%)$ & $(100 \%)$ \\
\hline Total & 159 & $131 \%)$ & 292 & 582 \\
& $(27,3 \%)$ & $(22,5 \%)$ & $(50,2 \%)$ & $(100 \%)$ \\
\hline
\end{tabular}

Ce tableau fait apparaître des traits communs avec d'autres régions : les pourcentages des années 1440-1490 sont proches de ceux de $\mathrm{Ph}$. Wolff pour Toulouse ${ }^{35}$. Cependant, l'évolution séculaire n'est pas sans appeler quelques commentaires. Que la proportion des apprentis originaires de la ville même n'augmente pas n'est guère étonnant. Il y a un plafonnement du recrutement local, surtout dans des villes moyennes comme Beaugency, la population n'ayant pas crû de façon spectaculaire.

${ }^{34} \mathrm{~J}$ 'ai considéré certaines paroisses de la banlieue orléanaise (Saint-Laurent, Saint-Marc, Saint-Vincent) avec la ville même. En revanche, Olivet a été placé dans le groupe II. Darvoy et St-Denis-de-l'Hôtel ont été groupés avec Jargeau.

${ }^{35} \mathrm{P}$. Wolff, op. cit., p. 81 : provenance des apprentis de Toulouse $27 \%$, abords de la ville $17 \%$, reste du royaume $56 \%$ (essentiellement de langue d'oc). 
En revanche il y a doublement du pourcentage des migrants dans la seconde moitié du XV $\mathrm{XV}^{\mathrm{e}}$ siècle.

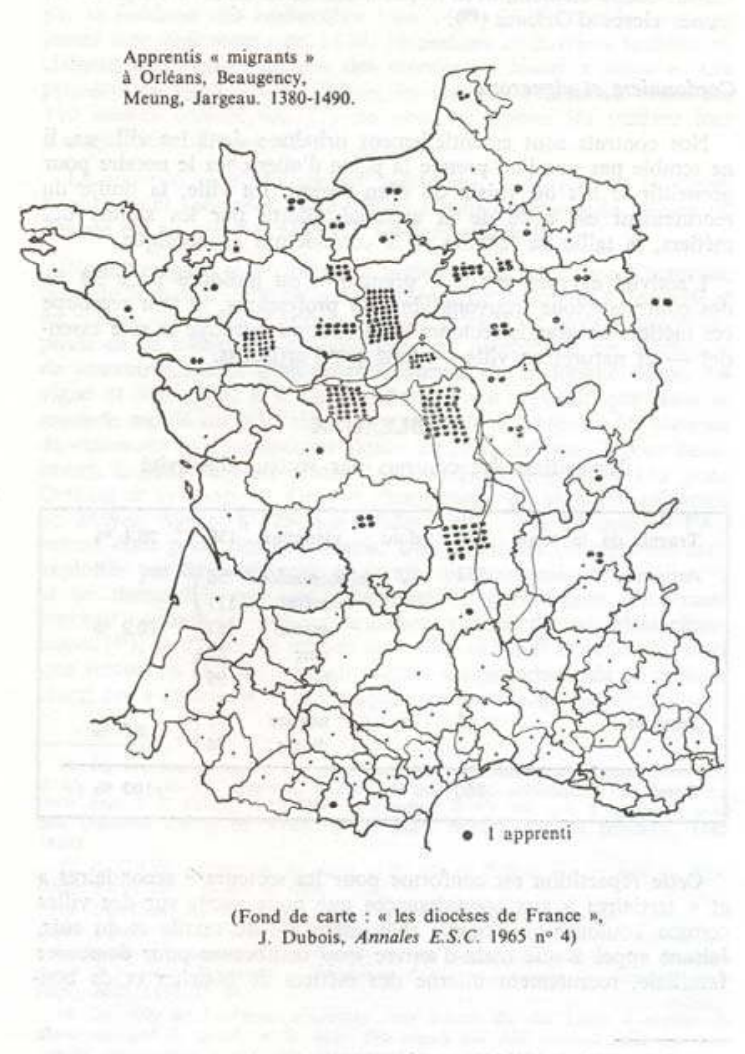

Il est certain que, dès l'unité du royaume reconstituée, des appels compensatoires de main-d'œuvre se firent de région à région, facilités par les grandes voies de communication. L'examen de la carte (Annexe 1) montre cependant que les déplacements lointains ont été rares. Les diocèses limitrophes de Chartres, Bourges et Tours sont les principaux fournisseurs. La Loire joue un rôle de canalisation des déplacements : du diocèse de Clermont, par l'Allier, on descend vers Orléans, tandis que les Angevins remontent le fleuve en s'arrêtant parfois à Beaugency ou Meung. La France d'oïl est le réservoir d'où sortent $95 \%$ des migrants; en effet, c'est du Bourbonnais que viennent principalement les jeunes gens qui quittent le diocèse de Clermont (ils viennent de Moulins ou de Gannat), Il n'y a donc pas d'obstacle de langue entre le patron et l'apprenti.

Ces migrants sont-ils isolés? On ne sera pas étonné de constater qu'ils forment $82 \%$ du groupe de ceux qui se présentent seuls. En y regardant de plus près, on repère quelques entrées en série : le 2 novembre 1464, trois jeunes gens nés près 
d'Angers se présentent chez trois vignerons orléanais ${ }^{36}$; le 20 août 1466 deux garçons du diocèse de Sées s'engagent chez deux peigneurs de laine associés ${ }^{37}$. On en voit venir chez un frère ${ }^{38}$, un cousin ${ }^{39}$. Mais ces cas sont rares, et nous n'avons aucun moyen de savoir s'il y a relation entre des apprentis venant de la même ville, ou de villages proches, et se retrouvant dans la même paroisse ligérienne.

Nous avons cependant un exemple inverse de déplacements liés les uns aux autres : Amy Hicas, receveur et grainetier du roi à Chalon-sur-Saône emmena avec lui, et à des dates différentes, plusieurs jeunes clercs d'Orléans ${ }^{40}$.

\section{Cordonniers et vignerons}

Nos contrats sont essentiellement urbains ; dans les villages, il ne semble pas que l'on prenne la peine d'aller chez le notaire pour accueillir le fils du voisin ou d'un parent. En ville, la limite du recrutement est celle de la capacité offerte par les statuts des métiers, la taille de la ville et la conjoncture économique. L'activité exercée par le preneur est indiquée pour $98 \%$ des contrats. Nous trouvons ainsi 86 professions. Si l'on regroupe ces métiers en grands secteurs d'activité, on retrouve le rôle essentiel - et naturel en ville - joué par l'artisanat.

TABLEAU X

Répartition des contrats par secteur d'activité

\begin{tabular}{|c|c|c|c|c|}
\hline $\begin{array}{l}\text { Travail de la } \\
\text { terre }\end{array}$ & 153 & & & $20,4 \%$ \\
\hline & & dont vignerons & 150 & \\
\hline Total Artisanat & 532 & & & $70,9 \%$ \\
\hline & & alimentation & 56 & \\
\hline & & métal & 111 & \\
\hline & & textile & 182 & \\
\hline & & cuir & 88 & \\
\hline & & bois & 95 & \\
\hline Total Services & 65 & & & $8,7 \%$ \\
\hline & & négoce & 11 & \\
\hline & & divers & 54 & \\
\hline Total & 750 & & & $100 \%$ \\
\hline
\end{tabular}

${ }^{36} 3$ E 10162 : Jehan Gohart, 19 ans, de St-Germain près d'Angers va à Ingré, Colas Bernard, 15 ans, de Louveux près d'Angers va à Saint-Vincent, Pierre Marignant, 16 ans, d'Angers va à Saint-Marceau.

${ }^{37} 3$ E 10164, 20-VIII-1466.

${ }^{38} 3$ E 10164, 29-XI-1466 : Guillaume Mynes, 17 ans, vient de Genillé (Indre-et-Loire) chez son frère Jean, teinturier à Orléans.

${ }^{39} 3$ E 10164, 24-X-1466 : Jean Beausse, 12 ans, de N.-D. de Tinchebray (Orne), est placé par son père chez Jehan Themiel, tondeur de grandes forces, son cousin. Le contrat ajoute : «l'intencion dudit Themiel n'est pas qu'il acquere avec soy aucun droit de communaulté ». ${ }^{40} 3$ E 10226, 24-VIII-1481 et 27-VIII-1481. 
Cette répartition est conforme pour les secteurs secondaire et tertiaire aux connaissances que nous avons sur des villes comme Toulouse ou Tours : rôle important du textile et du cuir, faisant appel à une main-d'œuvre trop nombreuse pour demeurer familiale, recrutement interne des métiers de boucher et de boulanger ${ }^{41}$. Certaines activités ne sont exercées qu'à Orléans : potier d'étain, armurier, poêlier, pelletier. Bien que la ville de Charles d'Orléans soit une des grandes villes du royaume, on est frappé par la faiblesse des embauches : en 1413, 23 tisserands en draps jurent leur règlement; en 1430, 36 maîtres et ouvriers foulons réclament une réglementation des ouvriers "allant à place». Les premiers ne figurent que 12 fois, les seconds 14 fois au cours des 110 années considérées. On ne peut qu'espérer les trouver lors d'autres dépouillements.

La surprise vient du secteur primaire. Le travail de la vigne, certes minutieux et spécialisé, différent du labeur ordinaire du paysan de Beauce, est au premier rang des métiers orléanais.

Nous touchons là un nouveau problème de fond de ces contrats, celui de la nature réelle de la contribution de l'apprenti à l'activité et au revenu du maître : apprenti ou/et valet? Le second intérêt du poids de ce métier est de nous montrer combien il est nécessaire de pouvoir disposer d'un corpus courant sur la longue durée. La vigne et son appel à l'apprentissage n'apparaissent que dans la seconde moitié du XV $\mathrm{XV}^{\mathrm{e}}$ siècle. Entre 1380 et 1440 , les 30 contrats de vignerons se décomposent ainsi : 20 pour Orléans, 2 pour Beaugency, 8 pour Jargeau. Entre 1440 et 1490, il y en a 119 pour Orléans et sa banlieue. On sait l'importance du vignoble orléanais au Moyen Âge et à l'époque moderne ${ }^{42}$ : vin de qualité, l'Auvernat était prisé des souverains. Une partie de ce vignoble était exploitée par des vignerons au service de bourgeois de la ville ${ }^{43}$ et ne demandait pas une main-d'œuvre permanente. Fortement éprouvé pendant les guerres anglaises, victime de calamités climatiques ${ }^{44}$, il n'a pu se relever qu'après 1435 . Il a nécessité alors une remise en état qu'il fallait assurer au moindre coût en embauchant des «apprentis ». Ces garçons ont certaines caractéristiques: ils sont relativement âgés (leur moyenne d'âge est de 17 ans), ils s'engagent pour 3 ou 4 années, ils perçoivent un salaire... En théorie, ils sont là pour apprendre «le fait de vignerie», mais pour l'un d'entre eux auquel le patron devra enseigner «le fait et stille du pressoer $»^{45}$, il s'en trouve dix qu'il "pourra loer et

${ }^{41}$ En 110 ans et dans l'état actuel du dépouillement et à Orléans seulement, il n’y a eu que 17 apprentis boulangers pour une soixantaine de boulangers (liste dans 3 E 10129, 17-IV-1406). De même il n'y eut que 6 apprentis pour une trentaine d'étaux de boucherie (A 2420, enquête sur les bouchers, 1440-1446).

${ }^{42} \mathrm{R}$. Dion, Histoire de la vigne et du vin en France des origines au XIX siècle, Paris, 1959, p. 272 sq.

${ }^{43}$ En 1380 , les habitants d'Olivet et de St-Hilaire-St-Mesmin refusent de payer l'impôt de la forteresse d'Orléans en disant qu'ils sont pauvres et que «les beaux heritaiges estans esdits paroisses ou la gragneure partie et le meilleurs estoit a plusieurs bourgois et habitants d'Orlians auxquels ne demouraient» $(\mathrm{BnF}, \mathrm{ms}$. fr. 1198, fol. 84).

${ }^{44}$ En 1406 les bourgeois d'Orléans vont auprès du duc Louis «exposer la grant pauvrete du peuple et la faulte des vignes qui deja avoient failli par trois annees enssuivantes » (CC 541 fol. $26 \mathrm{v})$.

${ }^{45} 3$ E $10212,2-X I-1468$. 
exploiter a son prouffit ou bon lui semblera es termes d'été $»^{46}$. Nous sommes vraiment à la limite de l'apprentissage et du louage de service au rabais ${ }^{47}$.

Les métiers féminins sont infiniment moins variés : couturières, lingères ou brodeuses ; les chambrières sont-elles autre chose que des servantes à tout faire, au pair?

L'accession à l'apprentissage d'un métier est-elle une forme d'ascension sociale ? Pour essayer d'en juger, nous ne disposons que de 201 contrats mentionnant la profession du père, soit un quart du total. Une trentaine de jeunes marche dans les traces paternelles : serrurier, potier d'étain, barbiers (deux cas), tisserands en toile (trois cas), et aussi vignerons (onze cas). Une vingtaine de familles au contraire manifeste dans le placement de leur fils une certaine ambition en payant pour le voir devenir «marchand», potier d'étain, mercier, alors que le père était laboureur. Des artisans et des marchands placent leur progéniture dans des bureaux de notaire ou mieux encore chez un receveur royal. Dans l'ensemble cependant, le corpus reflète une faible mobilité sociale: l'orfèvre place son fils chez le tailleur de monnaie, le manœuvre et le laboureur chez le vigneron, l'avocat chez le notaire, le marchand chez l'apothicaire ou le barbier. En somme la stabilité l'emporterait souvent, et nous ne percevons pas très nettement le désir de passer d'un métier de «labeur» à un métier «marchandant», marque d'un souci de promotion dans une certaine respectabilité. L'éventualité d'obtenir en fin d'apprentissage la franchise du métier ne figure qu'une seule fois, pour un tanneur. La tannerie était, à Orléans du moins, un métier « réglé ». Or aucun des neuf autres contrats de tanneur ne prévoit cette dispense. Cela ne veut nullement dire qu'en fin de service, l'apprenti ne pourra pas acheter le droit d'exercer après avoir prêté serment "par-devant les maîtres jurés»" ${ }^{48}$. Quant aux autres métiers sur lesquels nos actes sont muets, nous ne pouvons que constater notre ignorance.

\section{Le temps d'apprentissage : statuts et pratiques}

Les contrats prévoient la durée du séjour de l'apprenti chez le maître, le plus souvent à partir de la date de passation de l'acte. Il arrive que le point de départ soit fixé, pour le calcul des années, à partir d'une fête passée ou à venir : «d'aujourd'hui a la Saint-Jehan prouchainement venant et d'icelle Saint-Jehan à trois ans a venir» ou bien «de la Toussains derrenierement passee a deux ans a venir». On peut constater ainsi que le temps réel passé chez le maître peut dépasser un nombre rond

${ }^{46} 3$ E 10212, 25-III-1469 (n. st.). Cet acte ajoute «pourveu que ce soit avec gens de bien et ou il soit raisonnablement traité». Le terme d'été va de la Saint-Jean d'été à la Toussaint; au moment des vendanges on embauchait des journaliers que l'on payait mieux que les apprentis. Ceux-ci, peut-être, en profitaient-ils alors pour arrondir leur pécule.

${ }^{47} \ll$ Il n'y a pas de solution de continuité entre louage de service et apprentissage, celui-ci apparaît comme une simple modalité, la plus générale, du louage de service » (P. Didier, «Le critère de distinction entre louage de service et entreprise », Mémoires pour l'Histoire du droit et des institutions des anciens pays bourguignons, comtois et romands, $29^{\mathrm{e}}$ fasc., 1968-69, p. 209).

${ }^{48} \mathrm{~A} 2196$, fol. 20, règlement de 1388 . 
d'années ; on peut aussi se rendre compte que certains contrats ne sont que la légalisation postérieure d'accords verbaux.

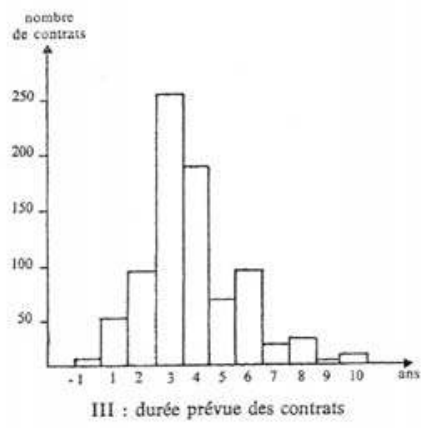

Théoriquement les métiers difficiles devraient exiger une plus longue préparation, tous ceux qui ont travaillé sur ce sujet sont d'accord pour dire qu'il n'en est rien $^{49}$. Le stage est essentiellement fonction de l'âge : plus on est jeune, plus il est long... Notre corpus étant surtout constitué d'adolescents majeurs, la durée de leur service est de 3 à 4 ans ${ }^{50}$.

Le mode, ici à 3 ans, est fortement accentué par le poids des contrats de vignerons. Néanmoins, même en enlevant les 150 contrats de 3 et 4 ans de ces apprentis, la moyenne de la durée prévue lors de l'établissement de l'acte est de trois ans et demi. Les 29 cas d'engagement à court terme (un an ou moins) ne présentent pas de caractéristique commune. Il peut s'agir de règlement de dette en travail ${ }^{51}$, du perfectionnement d'un fils auprès d'un collègue ${ }^{52}$, ou même d'un contrat à «l'envers », l'apprenti enseignant à son maître le métier qu'il pratiquait auparavant ${ }^{53}$.

Les statuts conservés des métiers d'Orléans ne prévoient pas tous la longueur du temps d'apprentissage, on ne peut donc vérifier systématiquement si la pratique suit le règlement. Les «tixiers en draps» et ceux en «toiles» exigeaient quatre ans : nous trouvons ce laps de temps observé 22 fois, mais à côté figurent des durées de 3 ans ( 2 cas), de 5 ans ( 4 cas), de 6 ans ( 6 cas), de 7 ans ( 2 cas), en tout 18 cas hors des normes. Les foulons demandaient quatre années et le paiement de 24 s. par. à la confrérie du métier: le temps est respecté, le paiement n'est prévu que dans un contrat sur deux. Ces deux exemples ne peuvent permettre une généralisation, mais l'extrême variété des situations laisse penser que les années passées près du maître étaient à la fois fonction de l'âge, des règlements, du rapport de force entre «bailleur», «alloué » et «preneur». La question mérite l'attention et un examen minutieux.

${ }^{49}$ Les potiers d'étain, par leurs statuts, prévoient 5 à 8 années d'apprentissage.

${ }^{50} \mathrm{G}$. Fagniez, op. cit., p. 58, écrit que la durée moyenne d'un contrat était de 6 ans, prudent, il ajoute «peut-être 3 ou 4 ».

${ }^{51} 3$ E $1179,17-I X-1446$.

${ }^{52} 3$ E 10233, 24-IX-1483 : le fils d'un tisserand d'Alençon.

${ }^{53} 3$ E 10211, 13-V-1467: l'apprenti enseignera à un "marchand» le métier de mégissiergantier. 
Le temps prévu était-il accompli? Seules les mentions marginales pourraient permettre de le dire, nous avons dit qu'elles sont rares. Nous avons connaissance de 39 ruptures ou fin de contrat. Les vraies ruptures (16 exemples) écourtent l'apprentissage : tel part au bout de quatre mois qui devait rester quatre ans ${ }^{54}$, un autre est renvoyé à la suite du décès du patron ${ }^{55}$, un troisième engagé, tout enfant, pour 11 ans, demande sa liberté au bout de 9 ans. Nous ne savons pas toujours les raisons qui président à ces séparations anticipées. Il ne s'agit pas de fuites, dans la mesure où on a pris soin d'en avertir le notaire et de préciser que les deux parties se «quittent l'une l'autre». Dans certains cas, nous avons la quittance de fin de service. On constate alors que des apprentis ont prolongé leur service : 5 ans et demi pour 5 ans, 6 ans pour 4,5 ans pour 3, 7 même pour 4 . Si certains contrats prévoient une récupération du temps de maladie en fin de service, ou un surcroît de durée pour compenser des pertes subies par le maître, il est à noter que cela ne correspond pas aux cas cités plus haut. Nous ne savons pas non plus si ces prolongations ont été salariées ou accomplies gratuitement.

\section{Payer et être payé}

Dans l'ensemble, les clauses financières et morales des contrats orléanais ne présentent aucune originalité : le preneur doit assurer l'entretien de celui qui se baille, sauf parfois les vêtements, exercer son métier honnêtement et montrer son «art». Nous retrouvons toujours cette même énumération : «il [le preneur] l'a pris pour être et demourer avec lui [...] et lui sera tenu querir et admenistrer, boire, mangier, feu, giste, hostel, sa chaussure de sollier». Les termes sont tout aussi vagues pour les obligations de l'apprenti : "servir du mieux qu'il pourra en toutes affaires licites, comme varlet apprantiz doit servir son maître». Rares finalement sont les clauses qui sortent de ce schéma, et prévoient la maladie ${ }^{56}$, la mort ${ }^{57}$, des travaux particuliers comme les embauches d'été des vignerons, le droit d'aller voir une mère veuve $^{58}$. Encore plus exceptionnelles sont les obligations imposées au maître de bien traiter «doulcement» un enfant ou de l'envoyer à l'école ${ }^{59}$, tout au plus devra-t-il le renvoyer parfois vêtu «comme il sied à son estat ».

\footnotetext{
${ }^{54} 3$ E $10226,6-$ III-1479 (n. st.).

${ }^{55} 3$ E 10136, 3-XI-1431 : la veuve renvoie l'apprenti. D'autres contrats montrent que parfois le successeur du maître garde l'apprenti : 3 E 10229, 3-X-1480, le garçon passe du père au fils.

${ }^{56} 3$ E 1180, 16-I-1448 : si l'apprenti [14 ans] «cheoit en maladie, ledit Aignan [un tisserand d'Orléans] le gardera jusqu'a un mois sans en rabattre rien de son service, mais s'il est malade plus d'un mois ledit Guillemin [l'apprenti] sera tenu de amender en la fin dudit temps ».

${ }_{57} 3 \mathrm{E} 10238,17-\mathrm{XI}-1488$ : le père ne paiera pas les 10 1.t. et le pourceau prévus si son fils meurt au cours de la première année.

${ }^{58} 3$ E 10224, 17-XI-1475 : contrat de Guillaume Porcher, 20 ans.

${ }^{59} 3$ E 10228, 3-V-1479 : régularisation d'un contrat oral passé deux ans auparavant. Michau Poyer, tonnelier, prend Guillaume Roulleau, 8 ans, « lequel il envoyera a l'escole jusqu'a ce qu'il ait appris son a b c et sept psaulmes, et lui fera donner tonsure, et [doit] icellui envoyer a l'escripture par l'espace de deux mois ». Au bout d'un contrat de 11 ans, il le renverra habillé et nanti d'une doloire et d'un compas.
} 
Les règlements financiers se présentent, comme ailleurs, sous deux formes : taxe versée au patron, salaire donné à l'apprenti. Cette seconde formule montre à l'évidence, pour ce présent corpus, que l'apprentissage peut n'être qu'une forme déguisée d'embauche pure et simple au taux le plus bas. Les versements sont en général prévus pour la fin du service, avec parfois des avances pour que le jeune homme puisse «querir ses nécessités », c'est-à-dire s'habiller. Ainsi est-il reconnu implicitement qu'un apprenti en fin de contrat peut réellement aider son maître et lui rapporter au lieu de lui coûter ${ }^{60}$. Ces salaires sont par ailleurs assez dérisoires, 2 livres-tournois par an en moyenne, mais, avec le vivre et le couvert, ils semblent suffisamment attrayants pour que des jeunes viennent d'assez loin les gagner. Les apprentis salariés sont proportionnellement plus nombreux dans la seconde moitié du $X^{\mathrm{e}}$ siècle et, bien entendu, le gros en est fourni par les vignerons (plus de la moitié).

TABLEAU XI

Clauses prévoyant un salaire (sur 821 contrats)

\begin{tabular}{|l|l|l|}
\hline & $1380-1440$ & $1440-1490$ \\
\hline Deniers ou blés & 110 & 217 \\
\hline Vêtements & 67 & 143 \\
\hline Outils & 23 & 51 \\
\hline Total & $200($ sur 377, soit $53,05 \%)$ & 411 (sur 444 soit $92,6 \%)$ \\
\hline
\end{tabular}

La médiocrité des salaires fait comprendre pourquoi les appoints en vêtement se font en même temps plus fréquents ; cependant il s'agit en général de rendre vêtu «convenablement», sans autres précisions, ou de fournir une paire de chausses par an; au mieux une robe pendant le temps du service; les jeunes vignerons reçoivent une ou deux «souquenyes", ce survêtement devant leur permettre de travailler sous la pluie ligérienne. Les maîtres ne sont guère plus généreux en outils : un cardeur donnera une paire de cardes ; un poêlier renverra au bout de quatre ans, bien vêtu et sans un sou, mais avec «pour le salaire des deux dernières années une paire d'outils telz qu'il appartient a un compagnon dudit metier», un garçon qui aura alors 23 ans $^{61}$.

Le versement par la famille de l'apprenti d'une somme d'argent ou la remise de biens en nature se trouve moins fréquemment qu'on ne le croit généralement ${ }^{62}$, et surtout nous voyons une désaffection pour cette formule au cours du $\mathrm{XV}^{\mathrm{e}}$ siècle, à la différence de ce que l'on peut voir, par exemple, dans la région de Montauban ${ }^{63}$.

${ }^{60}$ B. Geremek, op. cit., p. 52.

${ }^{61} 3$ E $10212,22-$ II-1468 (n. st.).

${ }^{62}$ A. Gouron, op. cit., p. 272 : « 1'apprenti donne très fréquemment une somme d'argent «.

${ }^{63} \mathrm{Ibid}$., p. 274, l'auteur voit s'accroître les versements au maître, mais il ne dispose que de 75 contrats. 
TABLEAU XII

Clauses prévoyant un versement au maître (sur 821 contrats)

\begin{tabular}{|l|l|l|}
\hline & $1380-1440$ & $1440-1490$ \\
\hline Deniers ou blés & 126 & 99 \\
\hline Travaux d'été & - & 23 \\
\hline Total & 126 & 122 \\
\hline
\end{tabular}

Cette évolution entre le début et la fin du siècle me conduit à revenir en partie sur ce que je pensais précédemment ${ }^{64}$. L'examen plus minutieux de ces versements montre bien que les orphelins sont relativement privilégiés dans la recherche d'un contrat, puisque le maître compte sur l'héritage et que les tuteurs, en payant ainsi, ne déboursent rien «du leur». Ce «privilège» qui gommait les clivages sociaux en rendant intéressants même de pauvres bougres, à condition qu'ils aient hérité ne serait-ce que d'une ruine ${ }^{65}$, s'estompe au cours du siècle. Les orphelins ne représentent plus que $46 \%$ des contrats payants contre $58 \%$ auparavant. En revanche, ce sont les fils de marchands d'Orléans, d'artisans aisés, de laboureurs de Beauce, garantis par des pleiges, qui sont accueillis contre versements du pécule au maître.

La monotonie que l'on ressent à la lecture de centaines de contrats n'est en fait qu'apparence. Devant la masse, la tentation première est de globaliser et de ramener tout à des schémas simples et rassurants. En étudiant de plus près, le chercheur est saisi par le doute. Il éprouve un doute quant à la forme "normale » de l'entrée dans la vie active par la médiation d'un contrat d'apprentissage, les orphelins sont privilégiés. Un second doute naît quant à l'âge «normal» de l'apprentissage à 14 ans, les contrats reflètent les ambiguïtés de l'âge de la majorité. En troisième lieu, l'ambivalence entre apprentissage et louage de bras paraît de plus en plus évidente. Enfin il n'est plus possible, me semble-t-il, de figer l'apprentissage en un tableau définitif et valable pour «le Moyen Âge»; dans la longue et même la moyenne durée, d'importantes variations surviennent en fonction des fluctuations économiques et démographiques générales et régionales.

\footnotetext{
${ }^{64}$ F. Michaud-Fréjaville, op. cit., p. 67.

${ }^{65} 2$ Mi 253, 22-XI-1429.
} 